\section{Terapia trombolítica en TEP submasivo/ riesgo intermedio: Evidencias y sugerencias post estudio Pulmonary Embolism Thrombolysis (PEITHO)}

\author{
ARNALDO MARÍN V. ${ }^{1}$, JOSÉ TOMÁS GAZMURI B. ${ }^{2}$, \\ MAX ANDRESEN V. ${ }^{\text {a }}$, MAX ANDRESEN ${ }^{3}$
}

\section{Thrombolytic therapy for submassive/ intermediate risk Pulmonary Embolism Evidence and suggestions after PEITHO trial}

Therapy for submassive pulmonary embolism (intermediate risk), remains controversial. New evidence has appeared that may help us in the process of decision making. We review the relevant literature, outline prognostic factors, and discuss current recommendations and controversies regarding the available alternatives such as systemic and catheter-directed thrombolytic use.

(Rev Med Chile 2015; 143: 895-904)

Key words: Pulmonary embolism; Shock; Thrombectomy; Thrombolytic therapy.
'Residente de Medicina Interna. Hospital Clínico de la Universidad de Chile. Facultad de Medicina, Universidad de Chile. Santiago, Chile.

${ }^{2}$ Residente de Medicina Interna. Facultad de Medicina Universidad de los Andes. Santiago, Chile.

${ }^{3}$ Departamento de Medicina Intensiva. Facultad de Medicina Pontificia Universidad Católica de Chile. Santiago, Chile.

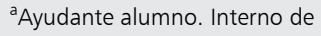
Medicina Facultad de Medicina Pontificia Universidad Católica de Chile.

Conflictos de intereses: ninguno que declarar.

Recibido el 20 de enero de 2015, aceptado el 2 de junio de 2015 .

Correspondencia a:

Dr. Max Andresen MsC

Departamento de Medicina Intensiva, Facultad de Medicina, Pontificia Universidad Católica de Chile.

Marcoleta 367, Santiago, Chile. andresen@med.puc.cl
$\mathrm{E}$ l espectro de presentación del tromboembolismo pulmonar (TEP) es amplio, desde casos asintomáticos hasta aquellos con severo compromiso hemodinámico, incluso muerte súbita. Las terapias en situaciones extremas: TEP leve, bajo riesgo y casos masivos (hipotensión mantenida o shock) no generan mayor controversia. Lo anterior contrasta con la controversia en casos de TEP submasivos.

En el año 2012 se inició un importante estudio: Pulmonary Embolism Thrombolysis trial (PEI$T H O)$, el cual evaluó el efecto de la trombolisis en pacientes normotensos, pero con disfunción ventricular derecha (VD). Los resultados fueron publicados en abril de 2014. Sin embargo, existe aún incertidumbre y se plantean críticas incluso luego de ser conocido en detalle.

El propósito del presente artículo es revisar las herramientas y estrategias para definir la población de pacientes con TEP submasivo que pueden beneficiarse de tratamientos más invasivos, en particular trombolíticos, a la luz de nueva evidencia. Para lo anterior se realizó una búsqueda amplia en PUBMED y EPISTEMONIKOS, respecto de TEP submasivo y trombolíticos, buscando estudios aleatorizados y revisiones sistemáticas, sin límites de fechas ni idiomas, abordando aspectos de efectividad en términos de recanalización, de mortalidad y riesgo de sangrado asociados a la terapia, buscando estudios que analizaran las variables que pudiesen incidir en este riesgo.

\section{Generalidades}

El TEP es una obstrucción mecánica de las arterias pulmonares (AP) asociada a vasoconstricción. Aumenta la resistencia vascular pulmonar y la postcarga del VD. Esta sobrecarga de presión puede generar hipoquinesia y dilatación del VD, insuficiencia tricuspídea $y$, en última instancia, insuficiencia VD y muerte.

Históricamente, la gravedad del TEP se enfocaba en el tamaño del trombo. Hoy reconocemos que depende del impacto hemodinámico, muy relacionado con el estado cardiopulmonar basal ${ }^{1}$. 
La American Heart Association clasifica el TEP 2 en tres categorías: masivo, submasivo y de bajo riesgo; la European Society of Cardiology ${ }^{3}$ en TEP de alto riesgo, riesgo intermedio y de bajo riesgo, considerando la presencia de shock o hipotensión, disfunción VD e injuria miocárdica.

Dado que la mortalidad del TEP puede ser mayor a $58 \%$ en pacientes en shock ${ }^{4}$, es en este escenario donde se recomienda el tratamiento trombolítico. En casos de TEP submasivo, que tiene mortalidad por recurrencia y además riesgo de desarrollar hipertensión pulmonar tromboembólica crónica (HPTC), podría ser útil la trombolisis $^{4-7}$. La trombolisis disminuye el riesgo de HPTC $^{8-10}$ al compararlo sólo con anticoagulantes.

Sin embargo, los estudios que emplean como único criterio la disfunción VD para decidir el uso de trombolíticos no han demostrado mejorar la sobrevida ${ }^{11}$. Sabemos que el grupo de pacientes con TEP submasivo es heterogéneo, por esto se ha intentado discriminar subgrupos de mayor riesgo, potencialmente beneficiarios de trombolisis. Es así como aparecen métodos de estratificación mediante biomarcadores y de gravedad de disfunción VD.

\section{Trombolíticos}

Estos reducen la resistencia y presiones pulmonares a las $2 \mathrm{~h}$ de iniciada la terapia, siendo útiles hasta las 2 semanas de síntomas ${ }^{12}$.

El empleo de trombolíticos no selectivos es limitado por sus efectos adversos. Por ello se prefieren agentes fibrino-selectivos, destacando el alteplase, muy estudiado y disponible, con reacciones adversas tolerables ${ }^{13-15}$. El resto se diferencia de alteplase. Así, reteplase posee mayor rapidez y duración de acción ${ }^{16}$, tenecteplase tiene bajo clearance y mayor especificidad por fibrina ${ }^{17-20}$, desmoteplase posee vida media más larga y es dos veces más potente ${ }^{21}$.

El riesgo de los trombolíticos son las hemorragias, con tasas de hemorragia mayor de $13 \%$ y letal de $1,8 \%{ }^{11,22-31}$. En pacientes inestables los trombolíticos reducen la mortalidad $(19,0 \%$ a 9,4\%) o recurrencia del TEP (OR, 0,45 ; IC de 95\%: $0,22-0,92)^{32}$, con respuesta favorable de $92 \%$ en las primeras $36 \mathrm{~h}^{33}$.

Los esquemas de tratamiento más frecuentes de trombolíticos se muestran en la Tabla 1 y las contraindicaciones para su empleo en la Tabla 2.

La controversia para el uso de los trombolíticos se centra en los pacientes hemodinámicamente estables $^{35}$. Su utilidad podría estar en pacientes con disfunción VD, la cual se presenta entre 31 y $64 \%$ de los pacientes ${ }^{36-39}$, con aumento de mortalidad de $66 \%$ respecto al TEP estable (OR: 2,53; IC a 95\% 1,17-5,50) $)^{35}$. Para identificar la disfunción VD se emplea:

\section{Ecocardiografía}

Se buscan alteraciones en motilidad, dilatación $\mathrm{VD}(\mathrm{VD} / \mathrm{VI}>0,9)$, septum aplanado y movimiento paradojal, HTP y, más tardíamente, insuficiencia

Tabla 1. Tipos de trombolíticos y su dosificación ${ }^{34}$

\begin{tabular}{|c|c|}
\hline Trombolíticos & Esquemas \\
\hline Estreptokinasa & $\begin{array}{l}\text { Carga de } 250.000 \mathrm{UI} \text { en } 30 \mathrm{~min} \text { y } 100.000 \mathrm{UI} \text { por hora sobre } 12-24 \mathrm{~h} \\
\text { Alternativa } 1,5 \text { millones de UI sobre } 2 \mathrm{~h}\end{array}$ \\
\hline Uroquinasa & $\begin{array}{l}\text { Carga de } 4.400 \mathrm{UI} \text { por } \mathrm{kg} \text { en } 10 \mathrm{~min} \text { y } 4.400 \mathrm{UI} / \mathrm{Kg} / \mathrm{h} \text { en } 12-24 \mathrm{~h} \\
\text { Alternativa: } 3 \text { millones UI en } 2 \text { o más horas }\end{array}$ \\
\hline Alteplase & $\begin{array}{l}100 \mathrm{mg} \text { en } 2 \mathrm{~h} \\
\text { Alternativa: } 0,6 \mathrm{mg} / \mathrm{kg} \text { en } 15 \mathrm{~min}\end{array}$ \\
\hline Reteplase & Dos bolos inyección de 10 UI en 30 min \\
\hline Tenecteplase & $\begin{array}{l}\text { Bolo de } 30-50 \mathrm{mg} \text { en 5-10 min Tabla de ajuste por kg de peso: } \\
<60 \mathrm{~kg}: 30 \mathrm{mg} \\
60-<70 \mathrm{~kg}: 35 \mathrm{mg} \\
70-<80 \mathrm{~kg}: 40 \mathrm{mg} \\
80-<90 \mathrm{~kg}: 45 \mathrm{mg} \\
>90 \mathrm{~kg}: 50 \mathrm{mg}\end{array}$ \\
\hline
\end{tabular}

*Los fabricantes recomiendan que los trombolíticos de nueva generación deberían usarse con HNF. 
Tabla 2. Contraindicaciones para el uso de trombolíticos ${ }^{3}$

\begin{tabular}{|ll|}
\hline Absolutas & Relativas \\
\hline Accidente cerebral vascular (ACV) hemorrágico o no conocido & TIA en los 6 meses previos \\
\hline ACV isquémico en los 6 meses previos & Uso de tratamiento anticoagulante \\
\hline Tumores del sistema nervioso central & Embarazo-posparto (primera semana) \\
\hline $\begin{array}{l}\text { Traumatismo cráneo-encefálico las 3 semanas previas, Trauma } \\
\text { mayor, cirugía }\end{array}$ & Sitios de punción no compresibles \\
& Resucitación cardiopulmonar traumática \\
& Hipertensión refractaria (PAS $>180 \mathrm{mmH})$ \\
& Enfermedad hepática avanzada \\
& Endocarditis infecciosa \\
\hline
\end{tabular}

triscupídea e hipocinesia VD. El principal problema es la falta de estandarización.

\section{Angio TAC pulmonar}

La razón $\mathrm{VD} / \mathrm{VI}>0,9$ identifica pacientes con mayor riesgo de mortalidad temprana ${ }^{36,39}$, no se cuenta con evaluación prospectiva de su utilidad para usar trombolisis. En nuestra experiencia, la razón VD/VI es indicador de la severidad del TEP. Hemos demostrado ${ }^{40}$ que se correlaciona significativamente con los valores de troponina y el score de Qanadli. Esto hace de la razón VD/VI un elemento rápido y asequible para estratificar el TEP.

\section{Biomarcadores}

Mejoran la sensibilidad de imágenes. Solos no deben ser empleados para definir conducta. Su incorporación en la definición de TEP intermedio permite estratificar en bajo o alto riesgo. Así, pacientes con biomarcadores positivos podrían beneficiarse de trombolisis si presentan disfunción VD. Respecto de péptidos natriuréticos (BNP $>90 \mathrm{pg} / \mathrm{mL}$ o pro BNP $>500 \mathrm{pg} / \mathrm{mL}$ ) se sabe que se asocian a mortalidad prematura (OR, 7,6; IC de $95 \%, 3,4-17)^{41}$. Troponinas: troponina I $>0,4$ $\mathrm{ng} / \mathrm{mL}$ o troponina $\mathrm{T}>0,1 \mathrm{ng} / \mathrm{mL}$ mayor mortalidad (OR, 5,24; IC de 95\%, 3,28-8,38) y eventos adversos (OR, 7,03; IC de 95\%, 2,42-20,43) en fase aguda ${ }^{42}$. En un estudio se encuentra proteína cardíaca de unión a los ácidos grasos ${ }^{43}$.

\section{Electrocardiograma}

Bloqueos, supra o infradesnivel de ST anteroseptal o inversión de onda $\mathrm{T}$ anteroseptal pueden indicar sobrecarga de presión de VD y orientar en la gravedad de la carga embólica.

Muchas sociedades recomiendan para la estratificación inicial el score de PESI (Pulmonary Embolism Severity Index, Tabla 3$)^{44}$.

La mortalidad intrahospitalaria del TEP submasivo es cercana a $2,5 \% \%^{4,6,7}$, de ahí que el diseño

Tabla 3. Score de PESI ${ }^{44}$

\begin{tabular}{|lc|}
\hline Variable & Puntaje \\
Demográfico & \\
Edad, por años & Edad, en años \\
Hombre & +10 \\
Comorbilidades & \\
Cáncer & +30 \\
ICC & +10 \\
Enfermedad pulmonar crónica & +10 \\
Hallazgos clínicos & \\
Taquicardia $>110$ & +20 \\
PAS $<100$ & +30 \\
FR $>30$ & +20 \\
Temperatura $<36^{\circ} \mathrm{C}$ & +20 \\
Alteración de conciencia & +60 \\
Saturación $<90 \%$ & +20 \\
Clasificación & \\
Clase I (muy bajo riesgo) & $<65$ \\
Clase II (bajo riesgo) & $66-85$ \\
Clase III (Riesgo intermedio) & $86-105$ \\
Clase IV (alto riesgo) & $106-125$ \\
Clase V (muy alto riesgo) & $>126$ \\
\hline
\end{tabular}


de estudios para evaluar la efectividad de trombolíticos en TEP submasivo reviste dificultades ${ }^{45}$, obligando a utilizar estrategias como grandes cohortes y outcomes compuestos para detectar diferencias significativas. Resultados secundarios, como disfunción persistente del VD, HTPC y deterioro de la calidad de vida, pueden representar objetivos para guiar la terapia en TEP submasivo.

Uno de los trabajos más importantes en esta área fue el desarrollado por el grupo de investigadores de Pulmonary Embolism Thrombolysis (PEI$\mathrm{THO})^{46}$, que fue un ensayo clínico aleatorizado, multicéntrico, con intención de tratar (1.005 pacientes). Compararon el uso de tenecteplase en dosis única vs anticoagulación con heparina no fraccionada (HNF) en TEP con signos de disfunción VD por ecografía o tomografía computarizada (TAC) y elevación de troponinas. Hubo reducción de la descompensación hemodinámica $(1,6 \%$ vs $5,0 \%, p=0,002)$ y diferencias en outcome primario combinado de descompensación hemodinámica y muerte a 7 días $(2,6 \%$ vs $5,6 \%, p=0,02, R R R$ $56 \%)$. Sin embargo, hubo diferencias en sangrado mayor, tanto intra como extracraneano $(6,3 \%$ vs $1,5 \%, p<0,001)$, más marcada en mujeres y en mayores de 75 años $(\mathrm{RRR}=37 \%)$. ACV cercano a $2 \%$, sin aumento de mortalidad en corto plazo.

Algunas dudas han surgido respecto al protocolo ${ }^{47}$ :

1. Trecientos tres pacientes que recibieron tenecteplase no recibieron anticoagulación con bolo de HNF o ésta fue diferida por haber recibido heparina de bajo peso molecular (HBPM) o fondaparinux en las 12 ó $24 \mathrm{~h}$ previas. Esto puede haber incidido en los outcomes, puesto que las HBPM tienen un efecto más lento. El haber utilizado HNF en todos podría mostrar un beneficio mayor en el grupo de pacientes que recibió tenecteplase.

2. Hay variabilidad en la respuesta a fibrinolíticos entre pacientes por diferencias en los antagonistas de activadores de plasminógeno (ej PAI-1), esto no fue considerado en el estudio. La variabilidad es mayor en personas mayores y condiciones inflamatorias asociadas.

3. No hubo evaluación centralizada ecocardiográfica de disfunción VD. Esto plantea dificultades por variabilidad interobservador.

4. Ausencia de estratificación clínica: en pacientes de bajo o muy bajo riesgo están contraindicados los trombolíticos.
5. Existen estudios que han utilizado dosis menores de trombolíticos en pacientes mayores de 75 años, tanto en IAM (tenecteplase) como TEP (alteplase) ${ }^{8}$. Esta pudiera ser una alternativa en pacientes mayores.

6. En $14,5 \%$ de los pacientes la disfunción VD fue sólo evaluada por TAC.

El estudio Tenecteplase or Placebo: Cardiopulmonary Outcomes at Three Months (TOP$\mathrm{COAT})^{48}$ intentó demostrar la utilidad de agregar tenecteplase en TEP submasivo. Además de buscar bajar mortalidad, intubación o shock, se diseñó un outcome compuesto a 3 meses del evento para evaluar morbilidad, considerando: nuevo TEP, funcionalidad cardiovascular $y$ percepción de salud. A diferencia del estudio PEITHO, utilizaron HBPM incluso en pacientes que ya recibían HNF.

A pesar de ser multicéntrico sólo reclutó 84 pacientes en 4 años; no demostraron diferencias en eventos adversos precoces (2,5\% vs $6,9 \%)$ tampoco los aspectos de la evaluación funcional a 3 meses, salvo una percepción general de salud que favorece el grupo que utilizó tenecteplase $(3,3 \text { vs } 2,4, p=0,036)^{48}$. El principal aporte del estudio TOPCOAT fue introducir la importancia de medir morbilidad a largo plazo como outcome clínico relevante.

El estudio abierto $\operatorname{MOPPET}^{9}(\mathrm{n}=121$ pacientes) con TEP moderado, definido por una oclusión trombótica de arterias lobar o principales $>$ a $70 \%$ en la TAC. El grupo de intervención recibió la mitad de dosis de trombolisis como bolo de alteplase de $50 \mathrm{mg}$; mostró reducción de HTP vs anticoagulación (RRA 40\% en 28 meses, 57\% vs $16 \%$ HTPC/TEP recurrente), sin aumentar el sangrado. A pesar de haber medido biomarcadores y disfunción VD, no fueron criterios para enrolar a los pacientes, empleando sólo criterios anatómicos, además de ser un estudio abierto y pequeño.

El estudio MAPPETT- $3^{11}$ fue en TEP e HTP o disfunción VD, sin hipotensión o shock. Utilizó HNF más 100 mg de alteplase o HNF más placebo durante $2 \mathrm{~h}$. El criterio principal de evaluación fue muerte intrahospitalaria o deterioro clínico que requiriera escalar en tratamientos. Sin diferencias en mortalidad intrahospitalaria $(3,4 \%$ vs $2,2 \%$, $\mathrm{p}=0,71$ ), hubo deterioro clínico que requirió escalada de tratamiento en los pacientes tratados 
con HNF sola $(24,6$ vs $10,2 \% ; p=0,004)$. Sin diferencias en incidencia de hemorragia mayor. Sólo 31\% de los pacientes tenían ecocardiograma con disfunción VD.

El registro multicéntrico de TEP (RIETE) ${ }^{49}$, con 15.944 pacientes, entregó información interesante. Pacientes normotensos mostraron riesgo de muerte asociado al trombolítico en comparación con pacientes que no la recibieron (OR 2,32; IC de $95 \%, 1,15-4,68 ; p=0,018)$. En otro registro $(\text { ICOPER })^{4}$ destaca hemorragia intracraneal en $3 \%$ vs $0,3 \%$ en casos que no recibieron trombolíticos, además de sangrado mayor en $22 \%$ vs $9 \%$, respectivamente.

Respecto de revisiones sistemáticas, la más actualizada $^{50}$ incluye 16 ensayos aleatorizados de trombolisis para TEP en general $(\mathrm{n}=2.115$; 4 estudios representan $74 \%$ de los pacientes) $37 \%$ de los pacientes fueron considerados hemodinámicamente estables con disfunción VD. Al comparar anticoagulación vs trombolisis se observó reducción en mortalidad general $(2,17 \%$ vs $3,89 \%$, OR 0,$53 ; 95 \%$ i IC $0,32-0,88$ ), con un número necesario para tratar relativamente bajo (NNT 59; 95\% IC 31-380, con RRA 1,12\%), con sangrado mayor más alto $(9,24 \%$ vs $3,42 \%$, OR $2,73 ; 95 \%$ IC; $1,91-3,91)$, con un número necesario para dañar (NNH 18; 95\%; IC13-27).

El análisis por subgrupos mostró que en pacientes mayores de 65 años no disminuye la mortalidad $(2,08 \%$ vs $3,65 \%$, OR $0,55,95 \%$ IC: $.29-1,05)$ y se mantiene el riesgo de sangrado mayor ( $12,9 \%$ vs $4,1 \%$, OR $3,10,95 \%$; IC: 2,10 4,56); en el subgrupo de menores de 65 años no se observó asociación con menor mortalidad o mayor sangrado. Considerando sólo pacientes con criterios de TEP submasivo ( $\mathrm{n}=1.775)$, se confirmó que el uso de trombolisis reduce la mortalidad ( $1,39 \%$ vs $2,92 \%$, OR $0,48,95 \%$; IC: $0,25-0,92)$, pero con más riesgo de sangrado mayor $(7,74 \%$ vs $2,25 \%$, OR $3,19,95 \%$ IC: 2,07 $4,92)$ al compararla con anticoagulación. Esta asociación se alcanza por los estudios de trombolisis para TEP submasivo conducidos entre 2009-2014 (estudios MAPPETT- $3^{11}$ y PEITHO ${ }^{46}$ respectivamente), que dan cuenta del tercio de los pacientes.

El metanalisis de Nakamura ${ }^{51}$ sólo incluyó estudios diseñados para TEP submasivo. La muestra consideró 6 estudios $(\mathrm{n}=1510)$, todos incluidos por Chatterjee ${ }^{50}$, sin embargo, mostró una re- ducción del outcome, compuesto de mortalidad general y deterioro clínico (3,9\% vs 9,4\%, RR 0,44, 95\% IC; 0,29-0,67), pero sin aumento del riesgo de hemorragia mayor (6,6\% vs $1,9 \%$, RR 2,07 ; 95\% IC: 0,58-7,35).

Un problema de los estudios es la definición inconsistente de TEP submasivo ${ }^{8}$ y dificultades en la estratificación.

En definitiva, la evidencia del uso de trombolíticos en TEP submasivo muestra:

1. A favor

a. Resolución sintomática más rápida.

b. Resolución de coágulos más rápida (reducción 30-35\% en el total de defectos de perfusión a las $24 \mathrm{~h}$ ).

c. Reducción temprana y a largo plazo de HTP.

d. Disminución de recurrencia del TEP.

e. Disminución del riesgo de inestabilidad hemodinámica.

2. En contra:

a. Riesgo de hemorragia mayor.

b. Mejoría similar a los 7 días, con o sin trombolisis.

c. Mayor costo.

Por todo esto es importante analizar variables que pueden incidir en el riesgo de hemorragia por trombolisis:

\section{a) Características del paciente}

1. Género: Un estudio ${ }^{52}$ muestra que la trombolisis vs anticoagulación en mujeres no tuvo menor mortalidad a 30 días $(6,3 \%$ vs $11,1 \%, \mathrm{p}=0,182)$, generó menor recurrencia del TEP $(8,3 \%$ vs $16,9 \%, p=0,05)$ y más sangrados mayores $(25 \%$ vs $8,4 \%, p=0,001)$. Además, respecto a la trombolisis en hombres, presentan más hemorragia intracraneal $(2,1 \%$ frente a $0 \%)$. Esto sugiere que los hombres pueden beneficiarse de trombolíticos en mayor grado que las mujeres.

2. Peso: Un análisis de subgrupos ${ }^{53}$ reveló que el riesgo de hemorragia fue mayor en pacientes con IMC menor de $24 \mathrm{~kg} / \mathrm{m}^{2}(42,9 \%$ vs $8,7 \%$, $\mathrm{p}=0,014$; RR 0,05; IC de 95\%: 0,01 a 0,57).

\section{b) Trombolítico}

1. Dosis: El trombolítco más utilizado es alteplase; la dosis recomendada en pacientes con 
peso mayor a $65 \mathrm{~kg}$ es un bolo de $10 \mathrm{mg}$ durante 1 a $2 \mathrm{~min}$, seguido de $90 \mathrm{mg}$ de infusión durante $2 \mathrm{~h}^{54}$. En pacientes con peso menor a $65 \mathrm{~kg}$, la dosis total administrada es de $1,5 \mathrm{mg} / \mathrm{kg}$. Dos estudios $^{53,55}$ muestran que en TEP submasivo, el usar la mitad de dosis no aumenta el sangrado y con una eficacia superior a la anticoagulación sola y similar respecto de dosis plena. Así, si la trombolisis está indicada, pero existe alto riesgo de sangrado, podría considerarse el uso de media dosis.

2. Bolo o infusión continua: La British Thoracic Society 2003 recomienda en el paro cardíaco por TEP administrar bolo de alteplase de $50 \mathrm{mg}^{13}$. La alteplase tiene vida media de 4 a $5 \mathrm{~min}$, infusiones mayores a $2 \mathrm{~h}$ logran concentraciones plasmáticas óptimas ${ }^{56}$. Un meta-análisis del año $2005^{57}$ evaluó publicaciones relacionados a infusión de alteplase, la dosis en bolo alteplase y estreptoquinasa. La infusión de alteplase fue más efectiva que la dosificación de bolo alteplase (RR 1,27; IC de 95\%: 1,09-1,47) con menores tasas de mortalidad debido al TEP (RR 0,16; IC de 95\%: 0,05 a 0,59) y un menor riesgo de hemorragia (RR 0,62; IC de 95\%: 0,43 hasta 0,96).

Así, en caso de estar indicada la terapia, el tratamiento es en infusión continua y se debe utilizar en media dosis del bolo en caso de paro, debido a la distribución limitada de la droga cuando la perfusión se encuentra comprometida ${ }^{28,29}$.

En definitiva, en TEP, para maximizar los beneficios de la trombolisis, se sugiere utilizar HNF con trombolítico ${ }^{28,30,58}$, reiniciando la infusión de HNF en pacientes con TEP submasivo cuando TTPa es menor que 60 a 80 segundos $^{59}$. Los pacientes mayores de 65-70 años, procedimientos invasivos recientes, presión arterial diastólica elevada (> $90 \mathrm{mmHg}$ ), peso menor $65 \mathrm{~kg}$ o IMC menor que 24, presentan mayor riesgo de sangrado a dosis estándar de trombolítico ${ }^{21,60-63}$.

\section{Otras terapias en TEP submasivo}

La administración de fibrinolíticos locales, embolectomía quirúrgica y la fragmentación o la aspiración del trombo ${ }^{64}$, podrían reducir el riesgo de hemorragia y ser igualmente eficaces. Nuestro equipo mostró con trombolisis directa ${ }^{65}$, que el NT-proBNP se correlacionó con reperfusión angiográfíca.
Una opción emergente es la trombolisis acelerada con ultrasonido (UAT), que requiere menos fibrinolíticos y así menos posibilidades de sangrado fatal.

ULTIMA $^{66}$ es un estudio aleatorizado de TEP submasivo $(\mathrm{n}=59)$ comparando HNF vs UAT de 10-20 mg activador del plasminógeno tisular recombinante de más de $15 \mathrm{~h}$. El outcome primario fue la diferencia en la relación de VD/ VI desde el inicio hasta las $24 \mathrm{~h}$. Los resultados de seguridad incluyeron mortalidad, decompensación hemodinámica, hemorragia grave y leve, TEP recurrente y los efectos adversos graves de hasta 90 días. En el grupo de UAT vs HNF, hubo una reducción significativa VD/VI $(0,30$ $\pm 0,20$ vs $0,03 \pm 0,16, p<0,001)$, a 90 días, no hubo diferencias en los eventos de sangrado ni recurrencias. Pareciera que UAT fue superior a HNF para aliviar la disfunción VD, sin un aumento en el sangrado. En definitiva por ahora el empleo de cada técnica dependerá del riesgo del paciente a sufrir un sangrado y de la experiencia y disponibilidad de unidades de radiología intervencional.

\section{Conclusiones}

El TEP submasivo corresponde a una entidad clínica que incluye una amplia gama de pacientes, con mortalidad y morbilidad a corto y largo plazo.

Desde el punto de vista fisiológico el TEP submasivo puede ser considerado como un estado temporal o de transición, dado que el estatus hemodinámico es un proceso dinámico y multifactorial, debiendo hospitalizarse los pacientes en unidades con monitoreo. Así, si el paciente empeora se pueda emplear trombolisis, con datos de efectividad hasta 14 días post TEP.

Con la información disponible no es posible recomendar la trombolisis a todos los pacientes con TEP submasivo. De manera que para obtener la máxima eficacia con el menor riesgo al indicar trombolisis es fundamental la estratificación de la disfunción VD (electrocardiograma, strain VD, biomarcadores), pudiendo considerarse la trombolisis en pacientes con TEP submasivo de riesgo intermedio alto, definido por el estudio PEITHO $^{46}$ (disfunción VD al ecocardiograma o TAC con troponina positiva) y con riesgo de sangrado tolerable (Tabla 4). 
Tabla 4. Resumen de Recomendaciones dadas por Sociedades Médicas respecto a Trombolisis en TEP

\begin{tabular}{|c|c|c|c|c|}
\hline Categoría & $\begin{array}{l}\text { British Thoracic } \\
\text { Society }^{13}\end{array}$ & $\begin{array}{l}\text { American College } \\
\text { of Chest Physician }{ }^{14}\end{array}$ & $\begin{array}{l}\text { European Society } \\
\text { of Cardiology Task } \\
\text { Force }^{3}\end{array}$ & $\begin{array}{l}\text { European Society of } \\
\text { Cardiology y European } \\
\text { Respiratory Society }{ }^{67}\end{array}$ \\
\hline Estratificar & $\begin{array}{l}\text { Sin mención } \\
\text { específica }\end{array}$ & Sí (IC) & Sin mención específica & $\begin{array}{l}\text { Si con Score PESI, si etapa } \\
\text { (III/IV) usar ecocardiograma } \\
\text { y biomarcadores }\end{array}$ \\
\hline TEP masivo & $\begin{array}{l}\text { Recomendado siem- } \\
\text { pre evaluar contrain- } \\
\text { dicaciones (IB) }\end{array}$ & $\begin{array}{l}\text { Recomendado siempre } \\
\text { evaluar contraindicacio- } \\
\text { nes (IB) }\end{array}$ & $\begin{array}{l}\text { Recomendado siempre } \\
\text { evaluar contraindica- } \\
\text { ciones }\end{array}$ & $\begin{array}{l}\text { Recomendado siempre eva- } \\
\text { luar contraindicaciones (IB) }\end{array}$ \\
\hline TEP submasivo & $\begin{array}{l}\text { No de primera línea } \\
\text { (IIB) }\end{array}$ & $\begin{array}{l}\text { Recomendado en pa- } \\
\text { cientes de alto riesgo a } \\
\text { menos que exista con- } \\
\text { traindicación (IIB) }\end{array}$ & No recomendado & $\begin{array}{l}\text { En pacientes de riesgo in- } \\
\text { termedio alto, considerar } \\
\text { trombolisis de rescate (IlaB) }\end{array}$ \\
\hline TEP estable & No recomendado & $\begin{array}{l}\text { No para la mayoría de } \\
\text { los pacientes (IB) }\end{array}$ & No mencionado & No recomendado \\
\hline Trombolítico & Alteplase (C) & Alteplase (IB) & Sin mención específica & $\begin{array}{l}\text { Sin mención por alguno en } \\
\text { particular }\end{array}$ \\
\hline Heparina & HNF (C) & $\operatorname{HNF}(\mid A)$ & HNF & HNF \\
\hline
\end{tabular}

\section{Referencias}

1. Wood KE. Major pulmonary embolism: review of a pathophysiologic approach to the golden hour of hemodynamically significant pulmonary embolism. Chest 2002; 121 (3): 877-905.

2. Jaff MR, McMurtry MS, Archer SL, Cushman M, Goldenberg N, Goldhaber SZ, et al. Management of massive and submassive pulmonary embolism, iliofemoral deep vein thrombosis, and chronic thromboembolic pulmonary hypertension: a scientific statement from the American Heart Association. Circulation 2011; 123: 1788-830.

3. Torbicki A, Perrier A, Konstantinides S, Agnelli G, Galiè N, Pruszczyk P, et al. Guidelines on the diagnosis and management of acute pulmonary embolism: The Task Force for the Diagnosis and management of Acute Pulmonary Embolism of the European Society of Cardiology (ESC). Eu Heart J 2008; 29: 2276-315.

4. Goldhaber SZ, Visani L, De Rosa M. Acute pulmonary embolism: clinical outcomes in the international cooperative pulmonary embolism registry (ICOPER). Lancet 1999; 353: 1386-9.

5. Vanni S, Nazerian P, Pepe G, Baioni M, Risso M, Grifoni $G$, et al. Comparison of two prognostic models for acute pulmonary embolism: clinical vs right ventricular dysfunction-guided approach. J Thromb Haemost 2011; 9 (10): 1916-23.
6. Todd JL, Tapson VF. Thrombolytic therapy for acute pulmonary embolism. Chest 2009; 135: 1321-9.

7. Kreit JW. The impact of right ventricular dysfunction on the prognosis and therapy of normotensive patients with pulmonary embolism. Chest 2004; 125: 1539-45.

8. Zarrabi K, Zolghadrasli A, Ostovan MA, Azimifar A. Short-term results of retrograde pulmonary embolectomy in massive and submassive pulmonary embolism: a single-center study of 30 patients. Eur J Cardiothorac Surg 2011; 40: 890-3.

9. Sharifi M, Bay C, Skrocki L, Rahimi F, Mehdipour M. Moderate pulmonary embolism treated with thrombolysis. Am J Cardiol 2013; 111: 273-7.

10. Greelish JP, Leacche M, Solenkova NS, Ahmad RM, Byrne JG. Improved midterm outcomes for type A (central) pulmonary emboli treated surgically. J Thorac Cardiovasc Surg 2011; 142: 1423-9.

11. Konstantinides S, Geibel A, Heusel G, Heinrich F, Kasper W, et al. Management Strategies and Prognosis of Pulmonary Embolism-3 Trial Investigators. Heparin plus alteplase compared with heparin alone in patients with submassive pulmonary embolism. N Engl J Med 2002; 347 (15): 1143-50.

12. Dalla-Volta S, Palla A, Santolicandro A, Giuntini C, Pengo V, Visioli O, et al. PAIMS 2: alteplase combined with heparin versus heparin in the treatment of acute pulmonary embolism-plasminogen activator Italian multicenter study 2. J Am Coll Cardiol 1992; 20: 520-6. 
13. British Thoracic Society Standards of Care Committee Pulmonary Embolism Guideline Development Group. British Thoracic Society guidelines for the management of suspected acute pulmonary embolism. Thorax 2003; 58: $470-83$.

14. Kearon C, Kahn SR, Agnelli G, Goldhaber S, Raskob GE, Comerota AJ, et al. Antithrombotic therapy for venous thromboembolic disease. Chest 2008; 133 (suppl): S445-54.

15. Torbicki A, van Beek EJR, Charbonnier B. Guidelines on diagnosis and management of acute pulmonary embolism. Eur Heart J 2000; 21: 1301-36.

16. Tebbe U, Graf A, Kamke W, Zahn R, Forycki F, Kratzsch $\mathrm{G}$, et al. Hemodynamic effects of double bolus reteplase versus alteplase infusion in massive pulmonary embolism. Am.Heart J 1999; 138: 39-44.

17. Keyt BA, Paoni NF, Refino CJ, Berleau L, Nguyen $\mathrm{H}$, Chow A, et al. A faster-acting and more potent form of tissue plasminogen activator. Proc Natl Acad Sci USA 1994; 91: 3670-4.

18. Sze DY, Carey MBL, Razavi MK. Treatment of massive pulmonary embolus with catheter-directed tenecteplase. J Vasc Interv Radiol 2001; 12: 1456-7.

19. Caldicott D, Parasivam S, Harding J, Edwards N, Bochner F. Tenecteplase for massive pulmonary embolus. Resuscitation 2002; 55: 211-3.

20. Kline J, Hernández-Nino J, Jones AE. Tenecteplase to treat pulmonary embolism in the emergency department. J Thromb Thrombolysis 2007; 23: 101-5.

21. Tebbe U, Graf A, Bramlage P, Lechleitner P, Bode C, Riess FC, et al. Desmoteplase in acute massive pulmonary thromboembolism. Thromb Haemost 2009; 101: 557-62.

22. Pulido T, Aranda A, Zevallos MA, Bautista E, Martínez-Guerra ML, Santos LE, et al. Pulmonary embolism as a cause of death in patients with heart disease: an autopsy study. Chest 2006; 129 (5): 1282-7.

23. The urokinase pulmonary embolism trial. A national cooperative study. Circulation 1973; 47 (2 Suppl.): II1108.

24. Levine M, Hirsh J, Weitz J, Cruickshank M, Neemeh J, Turpie AG, et al. A randomized trial of a single bolus dosage regimen of recombinant tissue plasminogen activator in patients with acute pulmonary embolism. Chest 1990; 98 (6): 1473-9.

25. Kanter DS, Mikkola KM, Patel SR, Parker JA, Goldhaber SZ. Thrombolytic therapy for pulmonary embolism. Frequency of intracranial hemorrhage and associated risk factors. Chest 1997; 111 (5): 1241-5.

26. Sors H, Pacouret G, Azarian R, Smyth AR. Hemodynamic effects of bolus vs 2 -h infusion of alteplase in acute massive pulmonary embolism. A randomized controlled multicenter trial. Chest 1994; 106 (3): 712-7.

27. Goldhaber SZ, Kessler CM, Heit JA, Elliott CG, Friedenberg WR, Heiselman DE, et al. Recombinant tissue-type plasminogen activator versus a novel dosing regimen of urokinase in acute pulmonary embolism: a randomized controlled multicenter trial. J Am Coll Cardiol 1992; 20 (1): 24-30.

28. Meyer G, Sors H, Charbonnier B, Kasper W, Bassand JP, Kerr IH, et al. Effects of intravenous urokinase versus alteplase on total pulmonary resistance in acute massive pulmonary embolism: a European multicenter double-blind trial. The European cooperative study group for pulmonary embolism [see comments]. J Am Coll Cardiol 1992; 19 (2): 239-45.

29. Goldhaber SZ, Kessler CM, Heit J, Markis J, Sharma GV, Dawley D, et al. Randomised controlled trial of recombinant tissue plasminogen activator versus urokinase in the treatment of acute pulmonary embolism. Lancet 1988; 2 (8606): 293-8.

30. Verstraete M, Miller GA, Bounameaux H, Charbonnier B, Colle JP, Lecorf G, et al. Intravenous and intrapulmonary recombinant tissue-type plasminogen activator in the treatment of acute massive pulmonary embolism. Circulation 1988; 77 (2): 353-60.

31. Urokinase-streptokinase embolism trial. Phase 2 results. A cooperative study. J Am Med Assoc 1974; 229 (12): 1606-13.

32. Wan S, Quinlan DJ, Agnelli G, Eikelboom JW. Thrombolysis compared with heparin for the initial treatment of pulmonary embolism: a meta-analysis of the randomized controlled trials. Circulation 2004; 110 (6): 744-9.

33. Meneveau N, Seronde MF, Blonde MC, Legalery P, Didier-Petit K, Briand F, et al. Management of unsuccessful thrombolysis in acute massive pulmonary embolism. Chest 2006; 129 (4): 1043-50.

34. Konstantinides S. Clinical practice. Acute pulmonary embolism. N Engl J Med 2008; 359 (26): 2804-13.

35. Ten Wolde M, Sohne M, Quak E, Mac Gillavry MR, Buller HR. Pronostic value of echocardiographically assessed right ventricular dysfunction in patients with pulmonary embolism. Arch Intern Med 2004; 164: 1685-9.

36. Schoepf UJ, Kucher N, Kipfmueller F, Quiroz R, Costello P, Goldhaber SZ. Right ventricular enlargement on chest computed tomography: a predictor of early death in acute pulmonary embolism. Circulation 2004; 110 : 3276-80.

37. Goldhaber SZ. Thrombolytic therapy for patients with pulmonary embolism who are hemodynamically stable 
but have right ventricular dysfunction: pro. Arch Intern Med 2005; 165: 2197-9.

38. Grifoni S, Olivotto I, Cecchini P, Pieralli F, Camaiti A, Santoro G, et al. Short-term clinical outcome of patients with acute pulmonary embolism, normal blood pressure, and echocardiographic right ventricular dysfunction. Circulation 2000; 101: 2817-22.

39. Sánchez O, Trinquart L, Colombet I, Durieux P, Huisman MV, Chatellier G, et al. Prognostic value of right ventricular dysfunction in patients with haemodynamically stable pulmonary embolism: a systematic review. Eur Heart J. 2008.

40. Andresen M, Marín A, Gazmuri JT, Meneses L, Andresen MA, Castro R. CT load score in the evaluation of submassive pulmonary embolism: correlation with RV/ LV diameter ratio and serum troponins. Clin Cardiol 2014; 20 (7): 1591-5.

41. Klok FA, Mos IC, Huisman MV. Brain-type natriuretic peptide levels in the prediction of adverse outcome in patients with pulmonary embolism: a systematic review and meta-analysis. Am J Respir Crit Care Med 2008; 178 (4): 425-30.

42. Becattini C, Vedovati MC, Agnelli G. Prognostic value of troponins in acute pulmonary embolism: a meta-analysis. Circulation 2007; 116 (4): 427-33.

43. Storch J, Thumser AE. The fattyacid transport functionof fattyacid-binding proteins. Biochim Biophys Acta 2000; 1486 (1): 28-44.

44. Aujesky D, Obrosky DS, Stone RA, Auble TE, Perrier A, Cornuz J, et al. Derivation and validation of a prognostic model for pulmonary embolism. Am J Respir Crit Care Med 2005; 172: 1041-6.

45. Pollack CV, Schreiber D, Goldhaber SZ, Slattery D, Fanikos J, O’Neil BJ, et al. Clinical characteristics, management, and outcomes of patients diagnosed with acute pulmonary embolism in the emergency department: initial report of EMPEROR (Multicenter Emergency Medicine Pulmonary Embolism in the Real World Registry). J Am Coll Cardiol 2011; 57: 700-6.

46. Meyer G, Vicaut E, Danays T, Agnelli G, Becattini C, Beyer-Westendorf J, et al. Fibrinolysis for patients with intermediate-risk pulmonary embolism. N Engl J Med 2014; 370 (15): 1402-11.

47. Smulders Y, Kramers C, Letter N. Engl J Med 2014; 371 (6): 579-82.

48. Kline JA, Nordenholz KE, Courtney DM, Kabrhel C, Jones AE, Rondina MT, et al. Treatment of submassive pulmonary embolism with tenecteplase or placebo: cardiopulmonar outcomes at 3 months: multicenter double-blind, placebo-controlled randomized trial. J Thromb Haemost 2014; 12: 459-68.
49. Riera-Mestre A, Jiménez D, Muriel A, Lobo JL, Moores $\mathrm{L}$, Yusen RD, et al. RIETE investigators. Thrombolytic therapy and outcome of patients with an acute symptomatic pulmonary embolism. J Thromb Haemost 2012; 10 (5): 751-9.

50. Chatterjee S, Chakraborty A, Weinberg I, Kadakia M, Wilenski RL, Sardar P, et al. Thrombolysis for pulmonary embolism and risk of all-cause mortality, major bleeding, and intracraneal hemorrhage: a meta-analysis. JAMA 2014; 311 (23): 2414-21.

51. Nakamura S, Takano H, Kubota Y, Asai K, Shimizu W. Impact of the efficacy of thrombolytic therapy on the mortality of patients with acute submassive pulmonary embolism: a meta-analysis. J Thromb Haemost 2014; 12 (7): 1086-95.

52. Geibel A, Olschewski M, Zehender M, Wilsch M, Odening K, Heinrich F, et al. Possible gender related differences in the risk to benefit ratio of thrombolysis for acute submassive pulmonary embolism. Am J Cardiol 2007; 99: 103-7.

53. Wang C, Zhai Z, Yang Y, Wu Q, Cheng Z, Liang L, et al. Efficacy and safety of low dose recombinant tissue-type plasminogen activator for the treatment of acute pulmonary thromboembolism: a randomized, multicenter, controlled trial. Chest 2010; 137: 254-62.

54. Alteplase spc. http://www.medicines.org.uk/emc/document.aspx?documentId=308

55. Sharifi M, Bay C, Skrocki L, Rahnimi F, Mehdipour M. Moderate pulmonary embolism treated with thrombolysis (from the 'MOPETT' Trial). Am J Cardiol 2013; 111: 273-7.

56. Meyer G, Gisselbrecht M, Diehl JL, Journois D, Sors H. Incidence and predictors of major haemorrhagic complications from thrombolytic therapy in patients with massive pulmonary embolism. Am J Med 1998; 105: 472-7.

57. Capstick T, Henry MT. Efficacy of thrombolytic agents in the treatment of pulmonary embolism. Eur Respir J 2005; 26: 864-74.

58. Piazza G, Goldhaber SZ. Management of submassive pulmonary embolism. Circulation 2010; 122: 124-9.

59. Mikkola KM, Patel SR, Parker JA, Grodstein F, Goldhaber SZ. Increasing age is a major risk factor for hemorrhagic complications after pulmonary embolism thrombolysis. Am Heart J 1997; 134: 69-72.

59. Agnelli G, Becattini C, Kirschstein T. Thrombolysis vs heparin in the treatment of pulmonary embolism: a clinical outcome-based meta-analysis. Arch Intern Med 2002; 162: 2537-41.

60. Simoons ML, Maggioni AP, Knatterud G, Leimberger JD, de Jaegere P, van Domburg R, et al. Individual risk 
assessment for intracranial hemorrhage during thrombolytic therapy. Lancet 1993; 342: 1523-8.

61. Fengler BT, Brady WJ. Fibrinolytic therapy in pulmonary embolism: an evidence-based treatment algorithm. Am J Emer Med 2009; 27: 84-95.

62. Piazza G, Goldhaber SZ. Management of submassive pulmonary embolism. Circulation 2010; 122 (11): 11249.

63. Moskovitz J. "Ultrasound accelerated thrombolysis. A safe and effective, low-dose thrombolytic therapy for acute pulmonary embolism”. Vein Magazine, 2012.

64. Andresen M, González A, Mercado M, Díaz O, Mene- ses L, Fava M, et al. Natriuretic peptide type-B can be a marker of reperfusion in patients with pulmonary thromboembolism subjected to invasive treatment. In J Cardiovas Imaging 2012; 28; 3; 659-66.

65. Kucher N, Boekstegers P, Muller OJ, Kupatt C, Beyer-Westendorf J, Heitzer T, et al. Randomized, controlled trial of ultrasound-assisted catheter-directed thrombolysis for acute intermediate-risk pulmonary embolism. Circulation 2014; 129: 479-86.

66. Konstantinides S, Torbicki A. Management of pulmonary embolism: recent evidence and the new european guidelines. Eur Respir J 2014; 44: 1385-90. 